\begin{tabular}{|c|c|}
\hline Title: & Transient Thermal Model for Ball Bearings in Electrical Machines \\
\hline Authors: & Felix Hoffmann, Donatas Silys, Martin Doppelbauer \\
\hline Institute: & $\begin{array}{l}\text { Karlsruhe Institute of Technology (KIT) } \\
\text { Institute of Electrical Engineering (ETI) }\end{array}$ \\
\hline Type: & Conference Proceedings \\
\hline Published at: & $\begin{array}{l}2020 \text { International Conference on Electrical Machines (ICEM) } \\
\text { Publisher: IEEE } \\
\text { Year: } 2020 \\
\text { ISBN: } 978-1-7281-9945-0 \\
\text { Pages: } 1018-1024\end{array}$ \\
\hline Hyperlinks: & https://ieeexplore.ieee.org/document/9270718 \\
\hline
\end{tabular}

(c) 2019 IEEE. Personal use of this material is permitted. Permission from IEEE must be obtained for all other uses, in any current or future media, including reprinting/republishing this material for advertising or promotional purposes, creating new collective works, for resale or redistribution to servers or lists, or reuse of any copyrighted component of this work in other works. 


\title{
Transient Thermal Model for Ball Bearings in Electrical Machines
}

\author{
Felix Hoffmann, Donatas Silys, Martin Doppelbauer
}

\begin{abstract}
With the trend towards smaller and high powerdensity machines, the thermal behavior of the machine is becoming increasingly important. Besides the windings and the magnets in Permanent Magnet Synchronous Machines (PMSMs), also the bearings are one of the critical components. The rotation of the balls inside a ball bearing significantly influences the thermal behavior of a bearing itself. Approaches from literature neglecting the thermal ball resistance or keeping it constant over the whole speed range do not give an accurate temperature estimation in all speed ranges for electrical machines. Therefore, we present a novel modeling approach with a speed-dependent and speed-independent thermal ball resistance. Contact resistances, convection phenomena and the power loss inside the bearing were calculated as proposed in literature. A brief summary of calculating the losses and contact resistances in a ball bearing is presented. It is shown that the ball resistance decreases in exponential shape with an increasing rotational speed of the ball. The ball resistance is not dependent on the diameter of the ball, if it is normalized to its stationary value and a function of the circumferential speed. The speeddependent resistance equals the stationary resistance at about $280 \mathrm{~mm} \mathrm{~s}^{-1}$. With this approach, the temperature distribution of a ball bearing can be accurately determined over the entire speed range, whereas existing literature approaches are only accurate in either the low or high speed range.
\end{abstract}

Index Terms-ball bearings, electrical machines, transient modeling, lumped circuit analysis

\section{INTRODUCTION}

While designing high power-density electrical machines, it is essential to have a knowledge of the thermal behavior of the machine. Besides the windings and the magnets in Permanent Magnet Synchronous Machines (PMSMs), also the bearings are one of the most critical components. In the absence of the rotor cooling, most of the heat generated in the magnets and the rotor sheets needs to flow through the bearings before it is dissipated through the water jacket. As the stator has most likely a comparable or even higher temperature, the heat flux from the rotor across the air gap to the stator can be neglected. To model the transient behavior of ball bearings analytical lumped circuit analysis is used. A coupled structural-thermal numerical approach significantly

F. Hoffmann is with the Institute of Electrical Engineering (ETI), Karlsruhe Institute of Technology (KIT), Kaiserstr. 12, 76131 Karlsruhe, Germany (e-mail: felix.hoffmann@kit.edu).

D. Silys is with the Institute of Electrical Engineering (ETI), Karlsruhe Institute of Technology (KIT), Kaiserstr. 12, 76131 Karlsruhe, Germany (email: donatas.silys@student.kit.edu).

M. Doppelbauer is with the Institute of Electrical Engineering (ETI), Karlsruhe Institute of Technology (KIT), Kaiserstr. 12, 76131 Karlsruhe, Germany (e-mail: martin doppelbauer@kit.edu). raises the complexity and is therefore no option in the design process. To get a thermal behavior of the bearings in a reasonable time, a lumped circuit needs to be set up and parametrized. Therefore, the thermal resistances of the contacts between races and balls and the thermal resistance of the ball itself needs to be known precisely as well as the convection phenomena inside the bearing. The formation of the contact surfaces and therefore the contact resistances are well known in literature [1]-[3] likewise the losses in a ball bearing [4]. Furthermore, there are several approaches available to model the Dean flow [5], [6] and Taylor Couette flow [7], which are the dominant convection phenomena in ball bearings. For the conductive thermal resistance of the balls itself, the approaches in literature are limited. Nakajima [3] describes the thermal contact resistances depending on the acting forces on the bearing and validated them including the overall thermal resistance of the bearing on the test bench. He neglects the thermal ball resistance and takes the sum of both contact resistances as the only conductive heat path in the bearing. Isert [8] observed the heat transfer through a ball bearing at low angular velocities and assumes a constant thermal resistance of the balls. However, the calculations are only done at standstill. As the thermal behavior of the bearings at standstill or low angular velocities is not of high interest, a model for all rotational speeds is needed. Bossmanns [9] and Uhlmann [10] observed high speed spindles, which are operating in the speed range of electrical machines used in automotive applications. In their observations the conductive resistance through the ball is neglected. This leads especially at low speeds to an inaccurate estimation of the temperature behavior. A combination of the beforementioned approaches was analysed by Kylander [11]. He tested two ball bearings in electrical machines and set up a data regression for the overall thermal resistance of the bearing, which is linearly decreasing for increasing rotational speed of the inner ring. The problem is that this regression is limited to bearings with a mean diameter between $46 \mathrm{~mm}$ and $75 \mathrm{~mm}$. To come over the aforementioned problems, we are using numerical methods to identify the thermal resistance of the balls depending on their rotational speed. The thermal resistance of the balls is split up in a speed-independent and a speed-dependent part. Therefore, the conductive resistance of the ball bearing can be modeled accurate over the whole speed range leading to a more accurate temperature behavior of the bearing. In the following, a ball bearing used in electrical machines with a rotating inner ring is observed. 


\section{Thermal Modeling}

In the following section the different parts of a ball bearing will be analyzed and its main thermal resistances will be calculated to parametrize the lumped circuit.

\section{A. Power Loss}

For the transient modeling of a ball bearing the knowledge of the power loss in the bearing is essential. There are different friction effects, which contribute to the total power loss. The main effect is the friction between the balls and the races due to rolling and sliding of the balls. For bearings used in electrical machines for automotive applications, the friction between the possible sealing and the rings as well as the drag friction caused by an optional oil bath is neglected. The total power loss inside the bearing can be calculated as

$$
P_{\text {loss }}=\omega_{\mathrm{i}}\left(T_{\text {roll }}+T_{\text {slide }}\right),
$$

where $\omega_{\mathrm{i}}$ describes the angular velocity of the inner ring, $T_{\text {roll }}$ the rolling torque and $T_{\text {slide }}$ the sliding torque due to friction. Both friction torque components are calculated according to [4]. The rolling torque component can be described by

$$
T_{\text {roll }}=\phi_{\mathrm{ish}} \phi_{\mathrm{rs}} G_{\mathrm{rr}}(\nu n)^{0.6},
$$

where $\phi_{\text {ish }}$ describes the inlet shear heating reduction factor, $\phi_{\mathrm{rs}}$ the kinematic starvation reduction factor, $G_{\mathrm{rr}}$ a bearing type and load depending factor, $\nu$ the kinematic viscosity of the lubricant and $n$ the rotational speed of the inner ring. The sliding torque component can be expressed as

$$
T_{\text {slide }}=G_{\mathrm{sl}} \mu_{\mathrm{sl}} \text {. }
$$

Therein $\mu_{\mathrm{sl}}$ describes the sliding friction coefficient and $G_{\mathrm{sl}}$ a bearing type and load-dependent factor.

\section{B. Convection Coefficients}

Another important heat path from the inner ring to the outer ring is due to convection. In a ball bearing two phenomena occur, which will be described in the following. To have a better understanding of the variables, Fig. 1 shows a schematic of a ball bearing. The phenomena are the Dean flow, which describes the heat transfer between the races and

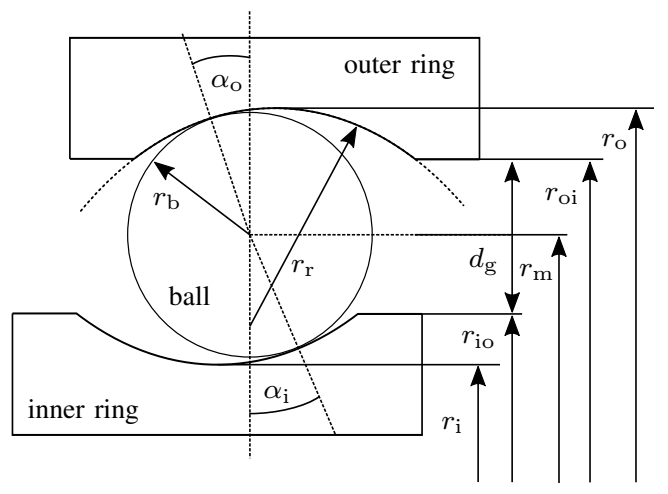

Fig. 1. Schematic of a ball bearing. the ball and the Taylor Couette flow, which describes the heat transfer between the rings. Both phenomena are speed dependent. The Dean flow, which occurs in curved channels where the radius of the curvature is much larger than the channel width, can be characterized by the Dean number [6]

$$
D e=R e_{\mathrm{De}}\left[\frac{d_{\mathrm{r}}}{2 r_{\mathrm{m}}}\right]^{1 / 2} .
$$

Therein $d_{\mathrm{r}}$ describes the raceway diameter and $r_{\mathrm{m}}$ the mean radius of the bearing. The Reynolds number for the Dean flow is defined by

$$
R e_{\mathrm{De}}=\frac{\omega_{\mathrm{i}} d_{\mathrm{b}}^{2}}{\nu},
$$

where $d_{\mathrm{b}}$ describes the ball diameter. Using the Dean number and the following Manlapaz-Churchill correlation [5], which is based on a regression analysis, the Nusselt number can be obtained as

$$
N u_{\text {De }}=\left[\left(3.657+\frac{4.343}{x_{1}}\right)^{3}+1.158\left(\frac{D e}{x_{2}}\right)^{3 / 2}\right]^{1 / 3}
$$

with $x_{1}=\left(1+\frac{957}{D e^{2} P r}\right)^{2}$ and $x_{2}=1+\frac{0.477}{P r}$, where $\operatorname{Pr}$ denotes the Prandtl number. Using (6), the heat transfer coefficient of the Dean flow can be calculated to

$$
\alpha_{\mathrm{De}}=\frac{N u_{\mathrm{De}} \lambda_{\mathrm{f}}}{d_{\mathrm{b}}}
$$

where $\lambda_{\mathrm{f}}$ describes the thermal conductivity of the lubricantair mixture.

For the Taylor Couette flow, we can directly calculate the Nusselt number according to [7]

$$
N u_{\mathrm{Ta}}=0.22\left(\frac{k R e_{\mathrm{Ta}}^{2}}{F_{\mathrm{g}}}\right)^{0.25} \operatorname{Pr}^{0.3} .
$$

Therein $k=\frac{d_{\mathrm{g}}}{r_{\mathrm{io}}}$ denotes the ratio between the gap length of the two races and the inner radius of the outer race and a geometrical factor defined by

$$
F_{\mathrm{g}}=\frac{0.05766(1+k / 2)}{0.0571(1-0.652 k)+0.00056(1-0.652 k)^{-1}} .
$$

The Reynolds number in this case is defined by

$$
R e_{\mathrm{Ta}}=\frac{\omega_{\mathrm{i}} r_{\mathrm{io}} d_{\mathrm{g}}}{\nu} .
$$

Using (8) and (10) we can directly calculate the heat transfer coefficient for the Taylor Couette flow

$$
\alpha_{\mathrm{Ta}}=\frac{N u_{\mathrm{Ta}} \lambda_{\mathrm{f}}}{d_{\mathrm{g}}} .
$$

\section{Contact Resistance}

If two curved surfaces are being physically pressed together, an elliptical contact zone is formed [2]. With the resulting contact surface, the contact resistance can be calculated, which is an essential part of the conductive heat path throughout a bearing. The resulting contact resistance is divided into two separate contact resistances, one at the inner 
and one at the outer contact area. They can be calculated for one ball according to [3] as follows

$$
R_{\mathrm{i}, \mathrm{o}}=\frac{\Psi\left(a_{\mathrm{i}, \mathrm{o}} / b_{\mathrm{i}, \mathrm{o}}\right)}{4 \lambda a_{\mathrm{i}, \mathrm{o}}}
$$

with $\Psi\left(a_{\mathrm{i}, \mathrm{o}} / b_{\mathrm{i}, \mathrm{o}}\right)=\frac{2}{\pi} K_{1}\left(e, \frac{\pi}{2}\right)$. Therein the indices $i$ and $o$ denote the inner and outer race, $\lambda$ the thermal conductivity of the bearing material, $a$ the semi-major half axis of the contact area, $b$ the semi-minor half axis, $K_{1}$ the complete elliptic integral of first kind and $e$ the eccentricity of the half axes. The total contact resistance of one ball assuming the same material for the ball and both races is given by [3]

$$
R_{\text {contact }}=\frac{1}{2 \lambda}\left[\frac{\Psi\left(a_{\mathrm{i}} / b_{\mathrm{i}}\right)}{a_{\mathrm{i}}}+\frac{\Psi\left(a_{\mathrm{o}} / b_{\mathrm{o}}\right)}{a_{\mathrm{o}}}\right] .
$$

The contact areas between the balls and the races are dependent on the axial and radial loads acting upon the bearing and must be calculated separately for each ball. The axial load can normally be neglected in comparison to the radial load in applications such as electrical machines, where radial ball bearings are used. Fig. 2 shows the distribution of the radial load $F_{\mathrm{r}}$ on the balls. The balls in the upper half are not loaded at all. The load on each ball can be calculated as

$$
F_{\mathrm{n}}=F_{\mathrm{r}} \cos ^{\frac{3}{2}}(n \theta), \quad \text { for } n \theta<\frac{\pi}{2} .
$$

Depending on the force on each ball, the contact areas and the thermal contact resistances are calculated. The contact resistance of the whole bearing is then given by the parallel connection of all contact resistances. In [2], a detailed derivation for calculating the contact surfaces can be found.

\section{Ball Rotation}

To model the transient thermal resistance of the balls inside the bearing, which changes due to the changing rotational speed, it is indispensable to know the angular velocity of the balls. The resulting formula for the rotational ball velocity calculation is shown in the following, whereas a detailed explanation can be found in [12]. The average angular velocity of the balls in dependency on the angular velocity of the inner ring is given by

$$
\omega_{\mathrm{b}}=-\frac{\omega_{\mathrm{i}}}{d_{b}} \frac{1}{\left(T_{1}+T_{2}\right)},
$$

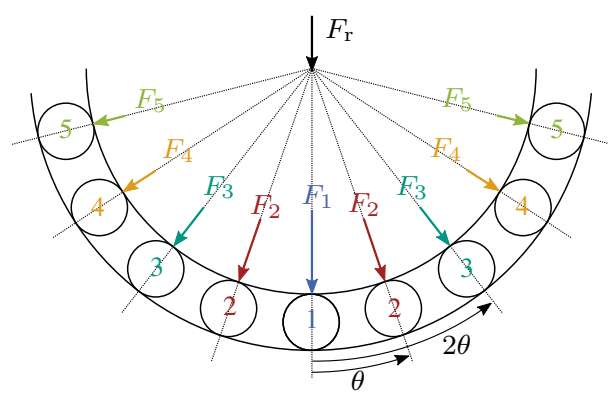

Fig. 2. Graphical representation of the radial load distribution on each ball.

$$
\begin{aligned}
\text { with } \quad T_{1} & =\frac{\cos \left(\alpha_{\mathrm{i}}-\arctan \left(\frac{\left.d_{\mathrm{m}} \sin \left(\alpha_{\mathrm{o}}\right)\right)}{d_{\mathrm{m}} \cos \left(\alpha_{\mathrm{o}}\right)+d_{\mathrm{b}}}\right)\right.}{d_{\mathrm{m}}-d_{\mathrm{d}} \cos \left(\alpha_{\mathrm{i}}\right)}, \\
\text { and } \quad T_{2} & =\frac{\cos \left(\alpha_{\mathrm{o}}-\arctan \left(\frac{\left.d_{\mathrm{m}} \sin \left(\alpha_{\mathrm{o}}\right)\right)}{d_{\mathrm{m}} \cos \left(\alpha_{\mathrm{o}}\right)+d_{\mathrm{b}}}\right)\right.}{d_{\mathrm{m}}+d_{\mathrm{b}} \cos \left(\alpha_{\mathrm{i}}\right)},
\end{aligned}
$$

where $d_{\mathrm{m}}$ denotes the mean bearing diameter. This implies that the ball is rotating in the opposite direction as the inner race. For the heat transfer it is indifferent in which direction the balls are rotating.

\section{E. Equivalent Circuit}

Fig. 3 shows the resulting equivalent circuit diagram for the ball bearing. The single ball shown in the figure represents all balls in the bearing. The previously discussed theory is applied to parametrize the resistances in the lumped circuit. The ball is split up in a speed-independent and a speeddependent thermal resistance. As the speed dependent one is difficult to calculate analytically, it is parametrized by Finite Element Analysis (FEA) simulations, which will be discussed in section IV-D. The contact resistances are calculated as stated in section II-C and the convection phenomena, described in section II-B, are combined into one thermal resistance. The conductive heat paths in the inner and outer races are not shown for the sake of simplicity. The cage is not modelled as it just holds the balls in its desired position and serves as an additional thermal mass, but does not contribute to the heat transfer between the two races. The generated heat is inserted with $50 \%$ to the ball and $25 \%$ to each contact region according to [11].

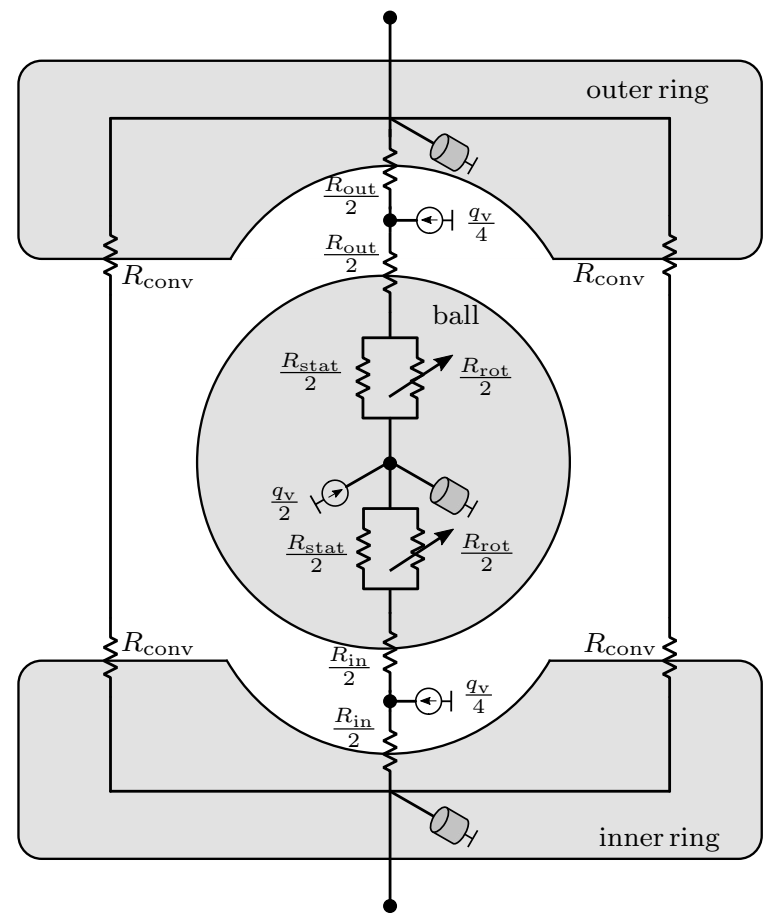

Fig. 3. Equivalent circuit of the ball bearing. 


\section{Device Under Test}

The observed bearing is a deep groove ball bearing of type $61807-2 \mathrm{RZ}$ from SKF [4]. This means that the bearing is fitted with two low frictional seals. Fig. 4 shows an graphical representation of the bearing.

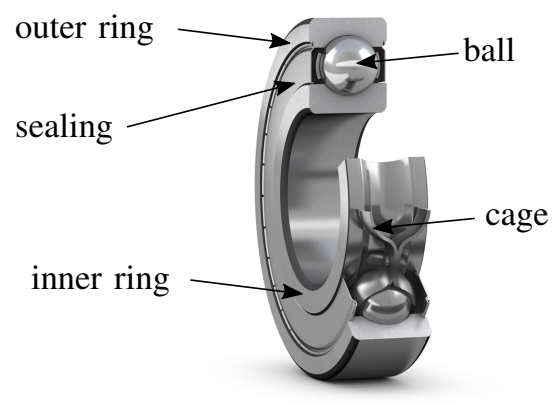

Fig. 4. Used ball bearing of type $61807-2 \mathrm{RZ}$ from SKF [4].

The ball bearing consists of 19 balls with a cage that holds the balls in position. The inner diameter of the inner race is given by $35 \mathrm{~mm}$ and the outer diameter of the outer race is given by $47 \mathrm{~mm}$. The bearing has a width of $7 \mathrm{~mm}$. The bearing is chosen for rotational speeds up to $15000 \mathrm{~min}^{-1}$ and is therefore applicable for automotive applications. The material of the balls and the races is stainless steel. The most important geometry parameters, thermal material characteristics and parameters for the analytical calculations are summarized in Table I. The thermal material characteristics are assumed to be not dependent on temperature.

TABLE I

BEARING PARAMETERS

\begin{tabular}{ccc}
\hline Parameters & \multicolumn{2}{c}{ Values } \\
\hline number of balls & 19 & \\
inner diameter & 35 & $\mathrm{~mm}$ \\
outer diameter & 47 & $\mathrm{~mm}$ \\
width & 7 & $\mathrm{~mm}$ \\
ball diameter & 3.5 & $\mathrm{~mm}$ \\
speed limit & 15000 & $\mathrm{~min}^{-1}$ \\
thermal conductivity & 60.5 & $\frac{\mathrm{W}}{\mathrm{mK}^{\mathrm{J}}}$ \\
specific heat capacity & 434 & $\frac{\mathrm{J}}{\mathrm{kg} \mathrm{K}}$ \\
volumetric mass density & 7850 & $\frac{\mathrm{kg}}{\mathrm{m}^{3}}$ \\
\hline
\end{tabular}

\section{Simulation Results}

In the following section, the lumped circuit parameters of the previous section are calculated in order to simulate the overall behavior of the ball bearing. For all simulations the bearing is loaded with a radial force of $500 \mathrm{~N}$.

\section{A. Power Loss}

The power loss of the machine is an essential parameter for modeling the bearing as the heat is directly inserted in the machine. Fig. 5 shows the result for the rolling and

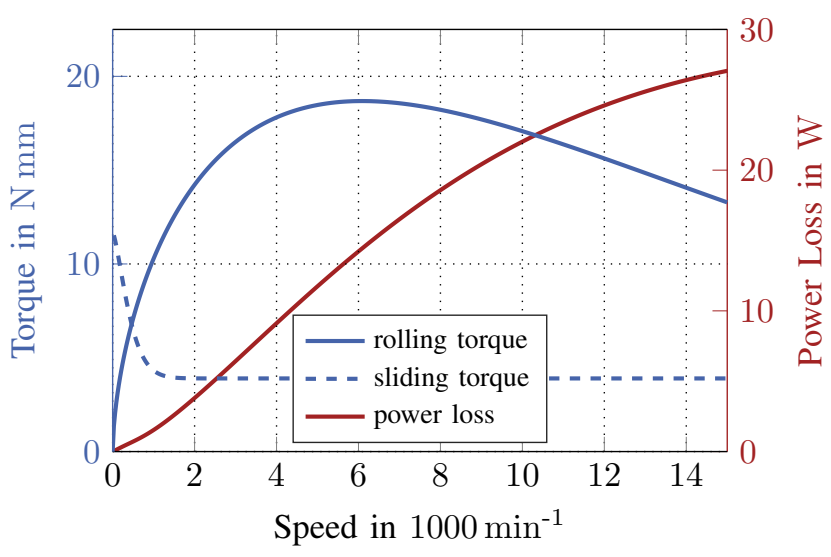

Fig. 5. Rolling torque and sliding torque due to friction (blue) and power loss (red) of the observed ball bearing at an operating temperature of $60^{\circ} \mathrm{C}$ for speeds of the inner ring up to $15000 \mathrm{~min}^{-1}$ and a radial load of $500 \mathrm{~N}$.

sliding torque as well as the overall power loss for speeds of the inner ring up to $15000 \mathrm{~min}^{-1}$. The power loss of a ball bearing is dependent on its operating temperature and therefore for the sake of simplicity just the results for an operating temperature of $60^{\circ} \mathrm{C}$ are displayed. The rolling torque is increasing up to about $18.7 \mathrm{~N} \mathrm{~mm}$ at $6000 \mathrm{~min}^{-1}$ and is decreasing afterwards in a linear shape, whereas the sliding torque is decreasing from $11.5 \mathrm{~N} \mathrm{~mm}$ down to $3.9 \mathrm{Nmm}$ at about $1800 \mathrm{~min}^{-1}$ and is staying constant afterwards. This is due to the fact that the lubrication is equally distributed at this point. Hence, the power loss is increasing from $0 \mathrm{~W}$ up to $27.1 \mathrm{~W}$ at $15000 \mathrm{~min}^{-1}$.

\section{B. Convetion Coefficients}

Fig. 6 shows the heat transfer coefficients of the Dean and Taylor Couette flow in dependency on the speed of the inner ring. It can be seen that both heat transfer coefficients are highly dependent on the rotational speed of the inner race. For low speeds up to $1000 \mathrm{~min}^{-1}$ a steep rise for the heat transfer coefficients can be seen, whereas the gradient is

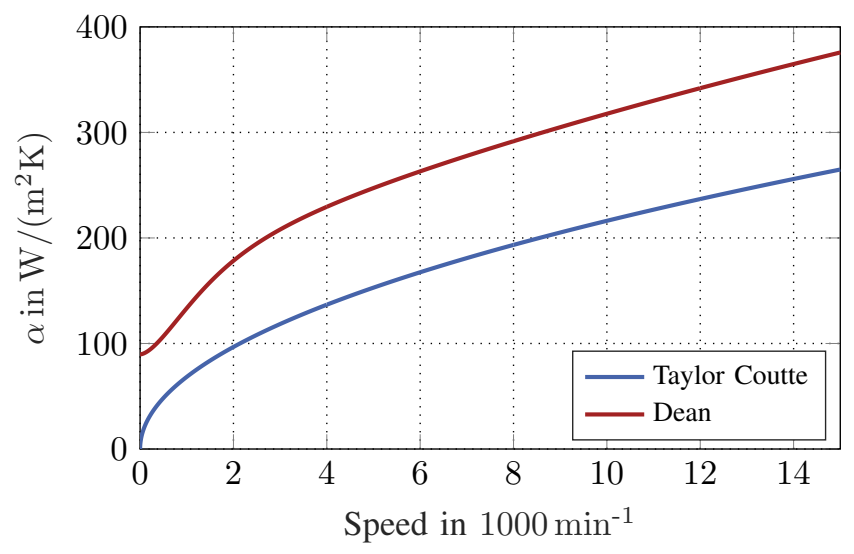

Fig. 6. Heat transfer coefficients of the Taylor Couette flow (blue) and the Dean flow (red) depending on the rotational speed of the inner ring. 
decreasing thereafter. This is due to the fact that the flow is laminar for low speeds and turbulent for high speeds, leading to an increasing heat transfer coefficient according to [5]. The heat transfer coefficient equals $376 \frac{\mathrm{W}}{\mathrm{m}^{2} \mathrm{~K}}$ at $15000 \mathrm{~min}^{-1}$ for the Dean flow and $265 \frac{\mathrm{W}}{\mathrm{m}^{2} \mathrm{~K}}$ for the Taylor Couette flow. The higher heat transfer coefficient for the Dean flow can be explained by a more turbulent flow between the race and the ball as the ball is rotating and the area where the vortices are forming is comparable small. Both effects lead to a higher heat transfer coefficient in comparison to the Taylor Couette flow, where the area between the inner ring and outer ring is comparable large and the outer ring is not rotating.

\section{Contact Resistance}

In radial ball bearings used in electrical machines the axial load is low compared to the radial load. Thus, only the sum of both contact resistances depending on the radial load is observed. Fig. 7 shows the contact resistance of the loaded balls. The number of the balls equals the one in Fig. 2. The contact resistance of the unloaded balls is infinite large and can be neglected. The contact resistance is decreasing with an increasing load as the ball is pressed more into the raceway. Thus, the contact surface between the ball and the ring is increasing. The same applies for the different balls. The ball, where the radial load vector is pointing to, has a much higher load and therefore a larger contact surface resulting in a lower thermal contact resistance. The total contact resistance of the ball bearing results with the parallel connection of the contact resistance of each ball. For the lumped circuit the contact resistance of each ball is assumed to be constant over time. This can be assumed as the inner race is rotating and the load on every ball changes rapidly. Fig. 8 depicts the total contact resistance between the balls and the inner and outer race. The thermal contact resistance decreases in exponential shape from $4.54 \mathrm{~K} / \mathrm{W}$ and $4.72 \mathrm{~K} / \mathrm{W}$ for $100 \mathrm{~N}$ down to $1.67 \mathrm{~K} / \mathrm{W}$ and $1.74 \mathrm{~K} / \mathrm{W}$ for a radial load of $2000 \mathrm{~N}$ for the inner and outer ring. The contact resistance is decreasing for an increasing radial load for the same reason as explained before. The contact resistance of the inner ring is slightly

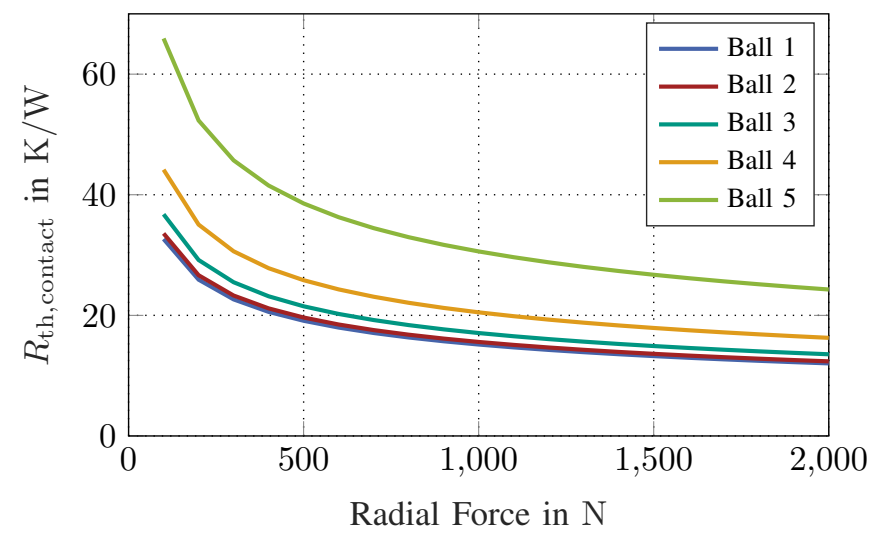

Fig. 7. Thermal contact resistance of the loaded balls of the inner ring dependent on the radial load.

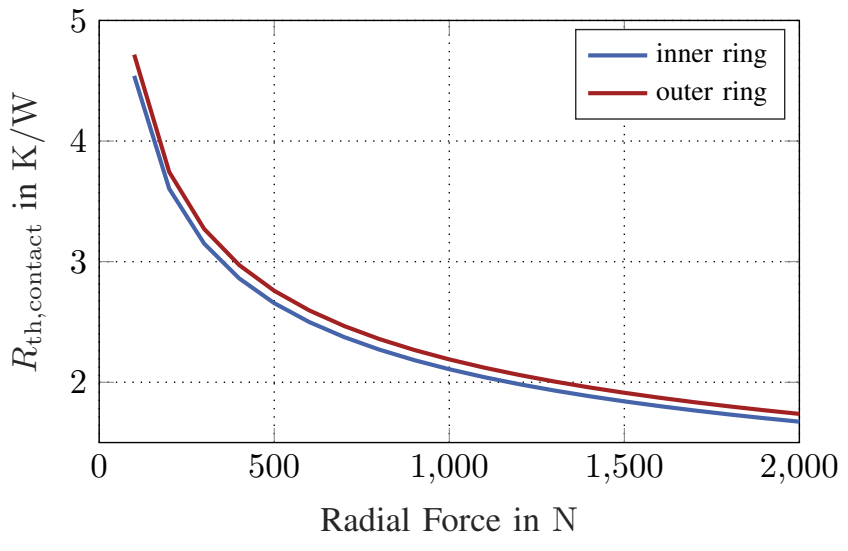

Fig. 8. Thermal contact resistance of the inner ring (blue) and outer ring (red) dependent on the radial load.

lower than the outer one as the contact surface is increasing the more curved the race is [2]. Since the inner ring is more curved, the contact resistance is lower. Hence, the greater the difference in the curvature of the races, the greater the difference in the thermal contact resistance.

\section{Ball Resistance}

The main improvement of the novel modeling of ball bearings is a changing conductive thermal resistance of the balls with changing rotational speed. Since analytical calculation methods are limited, FEA simulations are used to parameterize the resistance. Therefore, the thermal resistance depending on the rotational speed of the balls from 0 up to $50000 \mathrm{~min}^{-1}$ is simulated. The simulations were performed with ANSYS Mechanical. With section II-D it can be calculated that the rotational speed of the inner ring is 5.74 times lower than the one of the balls for the chosen load. Fig. 9 shows the result of the simulations. It is assumed that all balls are rotating with the same angular velocity due to the same reason as explained above for the averaging load. The thermal resistance of the ball is decreasing exponentially from

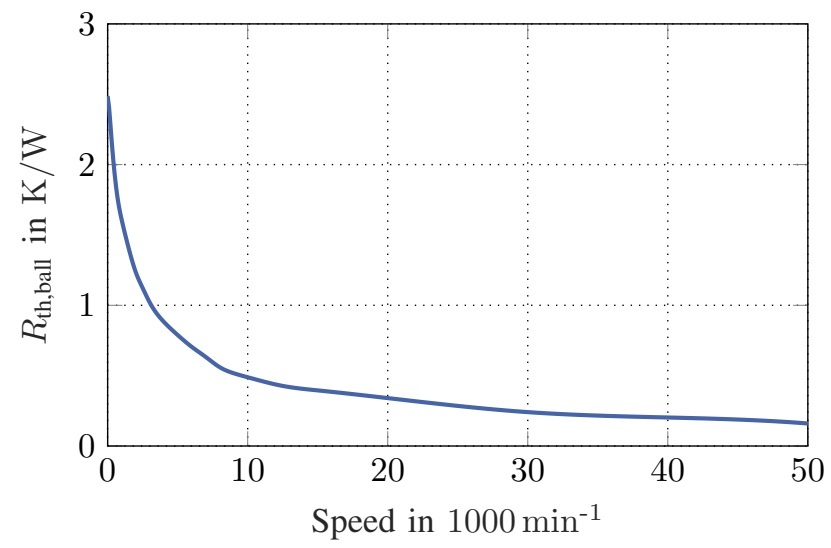

Fig. 9. Thermal resistance of all balls combined depending on the ball speed from standstill up to $50000 \mathrm{~min}^{-1}$. 
$2.5 \mathrm{~K} / \mathrm{W}$, which corresponds to its value at standstill, down to about $0.16 \mathrm{~K} / \mathrm{W}$ at $50000 \mathrm{~min}^{-1}$. The thermal resistance reaches $50 \%$ of its stationary value at about $2000 \mathrm{~min}^{-1}$. It can be seen that the behavior can not be described by a single exponential function, but a superposition of two exponential functions, as there are two effects happening for the heat transfer. The first one is the conductive heat path of the ball itself. Besides the conduction path, the heat will also flow from the inner race to the outer race due to the rotation of the ball. The heat is transported at the surface of the ball. The effect of the heat transfer due to rotation is increasing with increasing rotational speed and therefore the conductive ball resistance is converging to zero for infinite ball speed. Another important parameter for the conductive heat resistance of the rotating balls is the dependency on the ball diameter. To analyze this behavior equivalent simulations as before were performed. We will separate the speeddepending resistance part from the speed-independent part in the following. Fig. 10 shows the speed-dependent thermal resistance for different ball diameters normalized to their speed-independent resistance depending on the circumferential speed of the balls. The representation is chosen to have a better comparison between the balls. It can be seen that regardless of the ball diameter, the rotational thermal resistance depending on the circumferential speed is decreasing the same way. The differences in the ball diameters of up to $7 \%$ are due to simulation uncertainties of the mesh grid. The circumferential speed, where the two effects of heat transfer due to conduction due to rotation are equal is at about $280 \mathrm{~mm} \mathrm{~s}^{-1}$. Below this certain speed the conductive heat transfer is predominant, whereas the heat transfer due to the ball rotation is dominant beyond this point. Thus, the simulation results show that independent from the ball diameter, the rotational thermal resistance can be parametrized.

\section{E. System Simulation}

The lumped circuit is fully parametrized with the sections before and can be used to simulate scenarios, which are too

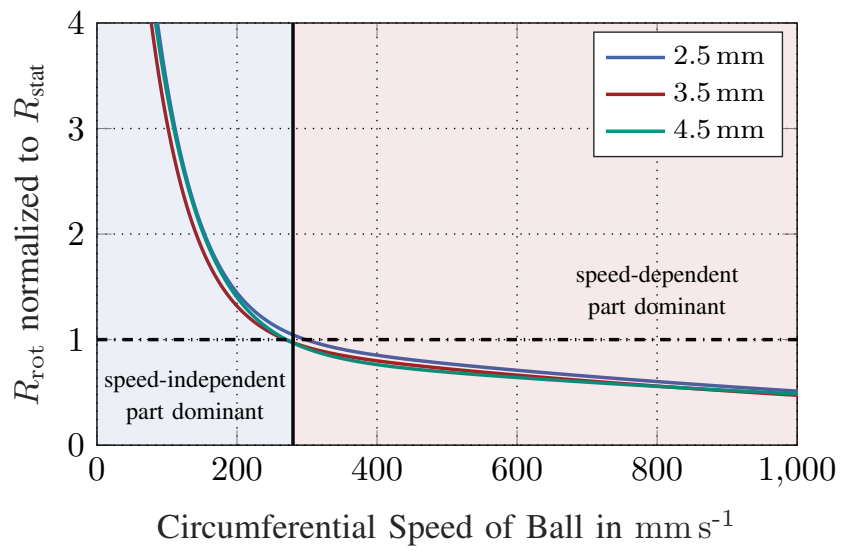

Fig. 10. Speed-independent thermal resistance of the balls normalized to its speed-independent resistance depending on the circumferential ball speed and ball diameters of $2.5 \mathrm{~mm}$ (blue), $3.5 \mathrm{~mm}$ (red) and $4.5 \mathrm{~mm}$ (green). complex to carry out in a coupled thermal-structural FEA simulation. The convection coefficients and heat loss inside the bearing will be calculated depending on the actual speed of the inner ring and the mean temperature of the bearing. A heat flux from the rotor of $10 \mathrm{~W}$ flowing through the bearing is assumed. To simulate the cooling, heat convection is assumed at the outer surface of the outer ring to a reference temperature of $50^{\circ} \mathrm{C}$.

The first simulation describes the temperature behavior of the ball bearing for a speed of the inner ring of $4000 \mathrm{~min}^{-1}$ until $150 \mathrm{~s}$. Thereafter, the speed is increased to $12000 \mathrm{~min}^{-1}$ until $300 \mathrm{~s}$ and is decreased to $6000 \mathrm{~min}^{-1}$ afterwards. Fig. 11 depicts the results. The temperature of the inner and outer ring as well as the one of the ball is increasing up to their steady-state values of $88.5^{\circ} \mathrm{C}, 76.3^{\circ} \mathrm{C}$ and $55.4^{\circ} \mathrm{C}$ at about $100 \mathrm{~s}$ for a speed of $4000 \mathrm{~min}^{-1}$. After the speed increases to $12000 \mathrm{~min}^{-1}$, the temperatures are increasing to $107.4^{\circ} \mathrm{C}, 97.2{ }^{\circ} \mathrm{C}$ and $60.6{ }^{\circ} \mathrm{C}$ in exponential shape. The temperatures are increasing as the power loss inside the machine is increasing and the decreasing ball resistance is not dominant in these speed ranges according to Fig. 9. For the same reason, the temperatures are decreasing again to $92.9^{\circ} \mathrm{C}, 81.4^{\circ} \mathrm{C}$ and $56.7^{\circ} \mathrm{C}$ after the speed decreases to $6000 \mathrm{~min}^{-1}$. The outer ring shows the coolest temperature as its thermal path to the cooling system is the shortest. On the contrary the inner ring is the hottest component as its thermal path is the longest. The temperature drop between the ball and the outer ring is larger compared to the temperature drop between the inner ring and the ball. This is due to the fact that the heat flux through the outer contact resistance is larger as it also consists of the heat losses of the bearing compared to the inner contact resistance. Due to the linear relationship between heat flux and temperature drop, the temperature drop is larger between the ball and the outer ring.

To see the importance of the novel modeling approach, the approaches from [8] and [10] are implemented and compared with each other for the same boundary conditions as before. Fig. 12 depicts the steady-state ball temperatures for a speed

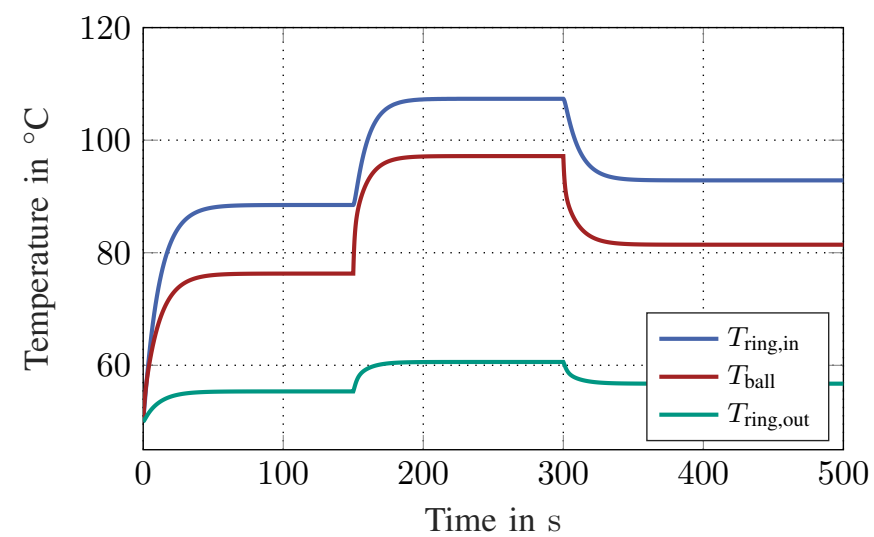

Fig. 11. Transient temperature behavior for the inner ring (blue), outer ring (green) and ball (red) for an radial load of $500 \mathrm{~N}$, a rotor flux of $10 \mathrm{~W}$ and speeds of $4000 \mathrm{~min}^{-1}, 12000 \mathrm{~min}^{-1}$ and $6000 \mathrm{~min}^{-1}$. 
of the inner ring from standstill up to $15000 \mathrm{~min}^{-1}$. It can be seen that the model, where the stationary ball resistance is kept constant over the speed range [8] starts at $86.8^{\circ} \mathrm{C}$ at standstill equivalent to our model. In comparison, the approach with neglecting conductive ball resistance [10] starts at $66.2^{\circ} \mathrm{C}$. This temperature difference of $20.6 \mathrm{~K}$ is directly given by the temperature drop over the thermal ball resistance. For increasing speeds the steady-state ball temperatures for the approach of a constant resistance are increasing, but the temperatures of our approach are decreasing up to $2000 \mathrm{~min}^{-1}$. This is due to the fact that the ball resistance is decreasing faster than the power loss inside the ball bearing is increasing with increasing speed. Our novel approach is converging to the approach with an neglected ball resistance and reaches it about $5500 \mathrm{~min}^{-1}$. At this speed the conductive ball resistance can be neglected and the approaches are reaching $104.4^{\circ} \mathrm{C}$ at $15000 \mathrm{~min}^{-1}$. The approach with a constant ball resistance is reaching $134.2^{\circ} \mathrm{C}$, which gives an overestimation of the actual temperature of $29.8 \mathrm{~K}$.

This simulation shows the importance of the novel approach to split up the thermal resistance of the ball in a speeddependent and speed-independent part. The approach with a constant ball resistance is just valid at standstill, but overestimates the temperature for higher speeds. On the other hand, the approach with neglecting ball resistance is just valid at high speeds, whereas our novel approach gives an accurate temperature estimation over the whole speed range. Especially in a use case, where the electrical machine stops and needs to accelerate afterwards, the heat flows from the rotor through the bearings, which would result in an underestimation of the actual temperature for a model with a neglected ball resistance.

\section{Conclusion And Outlook}

We have briefly discussed the contact resistances, convection phenomena and power loss inside a bearing. We have extended the thermal model of a ball bearing with a speeddependent and speed-independent thermal resistance for the

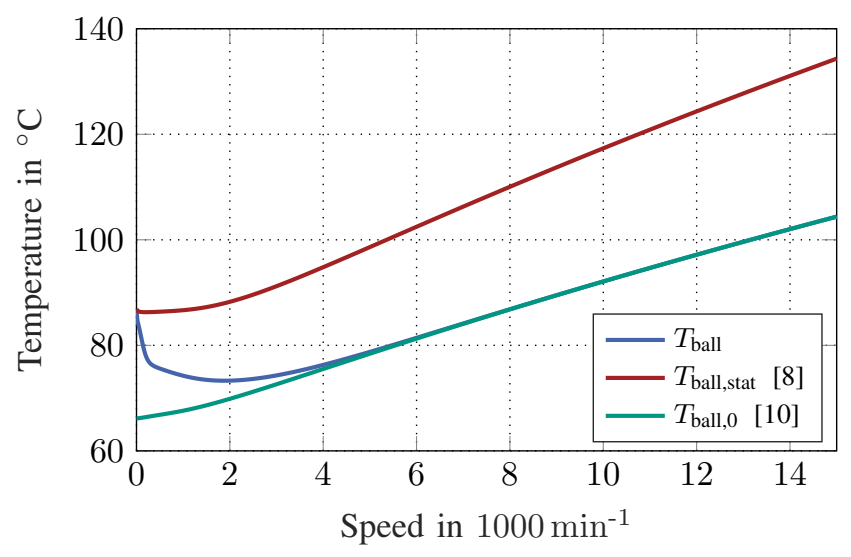

Fig. 12. Comparison of the steady-state ball temperatures over the whole speed range of the bearing for our approach (blue), a constant ball resistance (red) [8] and a neglected ball resistance (green) [10]. balls itself and have parametrized them with FEA simulations. Hence, we are able to simulate a speed-dependent heat transfer mechanism. Therefore, we are able to determine the temperature behavior precisely over the whole speed range. The total thermal resistance of the ball is decreasing in exponential shape and the speed-dependent and speedindependent part are equal for a circumferential speed of the balls of $280 \mathrm{~mm} \mathrm{~s}^{-1}$. It was shown that for the used ball bearing the steady-state temperatures of the balls over the speed range of the bearing is first decreasing until about $2000 \mathrm{~min}^{-1}$ and increasing afterwards. The novel approach equals the approach of a neglected ball resistance at about $5500 \mathrm{~min}^{-1}$. Currently we are setting up a test bench to measure the temperatures of the ball bearing to validate the results for the investigated ball bearing.

\section{REFERENCES}

[1] M. G. Cooper, B. B. Mikic, and M. M. Yovanovich, "Thermal contact conductance," International Journal of Heat and Mass Transfer, vol. 12 , no. 3, pp. 279-300, 1969.

[2] H. Nguyen-Schäfer, Computational Design of Rolling Bearings. Cham: Springer International Publishing, 2016.

[3] K. Nakajima, "Thermal contact resistance between balls and rings of a bearing under axial, radial, and combined loads," Journal of Thermophysics and Heat Transfer, vol. 9, no. 1, pp. 88-95, 1995.

[4] SKF, "Wälzlager," 2014.

[5] W. M. Rohsenow, J. P. Hartnett, and Y. I. Cho, Eds., Handbook of heat transfer, 3rd ed. New York: McGraw-Hill, 1998.

[6] Y. Chen, H. Chen, J. Zhang, and B. Zhang, "Viscoelastic flow in rotating curved pipes," Physics of Fluids, vol. 18, no. 8, 2006.

[7] A. Hiroshi, N. Hidetaka, and A. Hajime, "Convective heat transfer in an annulus with an inner rotating cylinder," The Japan Society of Mechanical Engineers, vol. 1967, no. 10, pp. 523-532, 1967.

[8] S. Isert, Heat Transfer Through A Rotating Ball Bearing At Low Angular Velocities. Utah State University, 2011.

[9] B. Bossmanns and J. F. Tu, "A thermal model for high speed motorized spindles," International Journal of Machine Tools \& Manufacture, vol. 39, pp. 1345-1366, 1999.

[10] E. Uhlmann and J. Hu, "Thermal modelling of a high speed motor spindle," Procedia CIRP, vol. 1, pp. 313-318, 2012.

[11] G. Kylander, Thermal modelling of small cage induction motors. Gothenburg: Chalmers Univ. of Technology, 1995.

[12] G. Jang and S.-W. Jeong, "Vibration analysis of a rotating system due to the effect of ball bearing waviness," Journal of Sound and Vibration, vol. 269 , no. 3-5, pp. 709-726, 2004.

\section{BIOGRAPHIES}

Felix Hoffmann was born in Edenkoben, Germany. He received his B.Sc. and M.Sc. degrees in electrical engineering from the Swiss Federal Institute of Technology Zurich (ETH Zurich), Zurich, Switzerland, in 2016 and 2018, respectively. He is currently working towards his Ph.D. degree at the Karlsruhe Institute of Technology (KIT), Karlsruhe, Germany, in the laboratory of hybrid electric vehicles (HEV). His research interests include the thermal analysis and thermal modeling of electrical machines.

Donatas Silys was born in Sirvintos, Lithuania. He received his B.Sc. degree in electrical engineering from the Karlsruhe Institute of Technology (KIT), Karlsruhe, Germany, in 2019. He is currently working towards his M.Sc. degree at the Karlsruhe Institute of Technology.

Martin Doppelbauer was born in Altenhundem, Germany. He received the Dipl.-Ing. and Dr.-Ing. degrees in electrical engineering from the University of Dortmund, Dortmund, Germany, in 1990 and 1995, respectively. He has worked with the companies Danfoss Bauer, Esslingen, and SEW Eurodrive, Bruchsal, Germany, as a Senior Manager for electric motor development. $\mathrm{He}$ has been holding the Professorship for hybrid electric vehicles at the Karlsruhe Institute of Technology, Karlsruhe, Germany, since 2011. 